\title{
Nuclear import factors importin $\alpha$ and importin $\beta$ undergo mutually induced conformational changes upon association
}

\author{
Gino Cingolani ${ }^{\mathrm{a}, \mathrm{c}}$, Hilal A. Lashuel ${ }^{\mathrm{b}}$, Larry Gerace ${ }^{\mathrm{a}}$, Christoph W. Müller ${ }^{\mathrm{c}, *}$ \\ ${ }^{a}$ Department of Cell and Molecular Biology, The Scripps Research Institute, La Jolla, CA 92037, USA \\ ${ }^{\mathrm{b}}$ The Skaggs Institute for Chemical Biology, La Jolla, CA 92037, USA \\ ${ }^{\mathrm{c}}$ European Molecular Biology Laboratory (EMBL), Grenoble Outstation, clo ILL, P.O. Box 156, 38042 Grenoble Cedex 9, France
}

Received 24 August 2000; revised 11 October 2000; accepted 12 October 2000

First published online 26 October 2000

Edited by Matti Saraste

\begin{abstract}
A heterodimer of importin $\alpha$ and importin $\beta$ accomplishes the nuclear import of proteins carrying classical nuclear localization signals (NLS). The interaction between the two import factors is mediated by the IBB domain of importin $\alpha$ and involves an extended recognition surface as shown by $\mathrm{X}$-ray crystallography. Using a combination of biochemical and biophysical techniques we have investigated the formation of the importin $\beta$ :IBB domain complex in solution. Our data suggest that upon binding to the IBB domain, importin $\beta$ adopts a compact, proteolytically resistant conformation, while simultaneously the IBB domain folds into an $\alpha$ helix. We suggest a model to describe how these dual mutually induced conformational changes may orchestrate the nuclear import of NLS cargo in vivo. (C) 2000 Federation of European Biochemical Societies. Published by Elsevier Science B.V. All rights reserved.
\end{abstract}

Key words: Nuclear import; Importin $\beta$; IBB domain; Conformational change

\section{Introduction}

Exchange of macromolecules between the cytoplasm and the nucleus occurs through the nuclear pore complex (NPC) and requires in most cases specific shuttling transport receptors $[1,2]$. The directionality of the movement through the NPC involves a cellular gradient of Ran, a small GTPase mainly bound to GTP in the nucleus and to GDP in the cytoplasm $[3,4]$. Transport receptors involved in nuclear import and export are related to importin $\beta$ (also known as karyopherin $\beta-1$ ) and form a family of proteins known as the importin $\beta$ super-family [5]. Import receptors bind their substrates in the cytosol, in the absence of RanGTP, whereas export receptors interact with their substrates in the nucleus in the presence of RanGTP.

Importin $\beta[6-8]$ is one of the most versatile transport receptors known in higher eukaryotes. It mediates the nuclear import of a large variety of substrates that interact with importin $\beta$ either directly or via adapters. Adapters characterized so far are importin $\alpha$, which recognizes classical nuclear localization signal (NLS) substrates [9-12], snurportin1 [13], RanBP7 [14] and XRIP $\alpha$ [15]. Importin $\alpha$ and snurportin 1 interact with importin $\beta$ via an IBB domain, an argininerich domain of roughly 40 residues $[16,17]$. Adapter independ-

*Corresponding author. Fax: (33)-476-20 7199.

E-mail: mueller@embl-grenoble.fr ent substrates can use IBB-like signals, like the HIV-1 proteins Rev and Tat [18], or lysine-rich domains, like the ribosomal protein L23a [19].

$\mathrm{X}$-ray crystallography has revealed the structures of importin $\beta$ bound to the IBB domain of importin $\alpha$ [20], a large $\mathrm{N}$ terminal fragment of importin $\beta$ bound either to RanGTP [21] or to five FxFG nucleoporin repeats from Nsplp [22] and yeast karyopherin $\beta 2$ (importin $\beta-2$, transportin) bound to RanGTP [23]. Both importin $\beta$ homologs are HEAT-repeat containing proteins. HEAT repeats are supersecondary structure motifs formed by two helices (named A and B helix) connected by a turn [24]. Importin $\beta$ contains 19 HEAT motifs, whereas 18 motifs have been identified in karyopherin $\beta 2$. The HEAT-repeats pack onto each other to form righthanded superhelical structures. Compared to the rather open conformation of karyopherin $\beta 2$, importin $\beta$ bound to the IBB domain of importin $\alpha$ has a more globular shape with repeats 7-19 wrapped tightly around the IBB domain. Here we report biochemical and biophysical evidence that the formation of the importin $\beta$ /importin $\alpha$ heterodimer leads to significant tertiary structure rearrangement in importin $\beta$ and to secondary structure formation in the IBB domain.

\section{Materials and methods}

\subsection{Protein expression and purification}

Human importin $\alpha$ and $\beta$, Ran, RanBP1 and HIV-1 Rev were expressed in Escherichia coli BL21(DE3) strain and purified as previously described $[17,25,26]$. Gel filtration was carried out on a Superdex-200 column (Pharmacia) using a BioRad fast protein liquid chromatography system. Samples were applied to a Superdex-200 column pre-equilibrated in $50 \mathrm{mM} \mathrm{NaCl}, 20 \mathrm{mM}$ HEPES pH 7.5, $5 \mathrm{mM}$ $\beta$-mercaptoethanol and separated at a constant flow rate of $0.25 \mathrm{ml} /$ min.

\subsection{Limited proteolysis}

Digestion reactions were performed at $37^{\circ} \mathrm{C}$ with a protease:protein ratio of $1: 250(\mathrm{w}: \mathrm{w})$ except for importin $\alpha$ shown in Fig. 3a, which was digested on ice for $12 \mathrm{~h}$ in a protease:protein ratio of $1: 1000$ (w:w). Reactions were started by the addition of freshly dissolved protease, and $10 \mu \mathrm{l}$ aliquots were withdrawn at regular intervals. Reactions were stopped by the addition of $1 \mu \mathrm{PMSF} 10 \mathrm{mM}$ and $10 \mu \mathrm{l}$ of sodium dodecyl sulfate (SDS)-protein sample buffer. Samples were boiled for 4 min and analyzed on SDS-polyacrylamide gel electrophoresis (PAGE) followed by Coomassie blue staining. Electrophoretic gels under native conditions were prepared as described [27].

\subsection{Fluorescence spectroscopy}

Steady state fluorescence spectra were acquired in a Perkin Elmer spectrofluorimeter. All spectra were measured at $20^{\circ} \mathrm{C}$ in a $0.5 \times 0.5$ $\mathrm{cm}$ path length cuvette, in a final volume of $300 \mu$. Protein and 
peptide stocks were diluted to $5-10 \mu \mathrm{M}$ in digestion buffer. Selective excitation of Trp was performed at $295 \mathrm{~nm}$ and fluorescence emission spectra were recorded between 300 and $365 \mathrm{~nm}$ using a $1 \mathrm{~nm}$ step size per integration time and a constant ordinate scale (in arbitrary units).

\subsection{Circular dichroism (CD)}

For CD, $20 \mu \mathrm{M}$ IBB domain was dissolved in $20 \mathrm{mM}$ HEPES $\mathrm{pH}$ 7.0 or $20 \mathrm{mM}$ potassium phosphate $\mathrm{pH}$ 4.2. Spectra were measured at $20^{\circ} \mathrm{C}$ between 190 and $250 \mathrm{~nm}$ with an AVIS spectrometer using a data point increment of $0.5 \mathrm{~nm}$. The signal to noise ratio was increased measuring three scans per spectrum: spectra were averaged and the buffer signal subtracted. 2,2,2-Trifluoroethanol (TFE) was added to 30 and $50 \%(\mathrm{w} / \mathrm{w})$. For thermal unfolding curves the ellipticity at $222 \mathrm{~nm}$ was monitored as a function of temperature between 20 and $80^{\circ} \mathrm{C}$. The final concentration of importin $\beta$ was $4.5 \mu \mathrm{M}$ whereas the importin $\beta$ :IBB domain was formed in a molar ratio of $1: 2$. The temperature was increased from 20 to $80^{\circ} \mathrm{C}$ in $1{ }^{\circ} \mathrm{C}$ increments followed by $1 \mathrm{~min}$ equilibration. Melting temperature $\left(T_{\mathrm{m}}\right)$ values were calculated after non-linear curve fitting of the data points had been performed.

\section{Results}

\subsection{Binding of the IBB domain induces a proteolytically resistant conformation of importin $\beta$}

Human importin $\beta$ was subjected to limited proteolysis using various proteases. Chymotrypsin cleaved most specifically and reproducibly, yielding a proteolytic fragment of approximately $50 \mathrm{kDa}$ (Fig. 1a, lane 2). N-terminal sequencing and mass spectrometry (data not shown) revealed that the cleavage occurred between residues 449 and 450, leading to the Cterminally truncated fragment $1-449$. Interestingly, in the presence of the adapter molecule importin $\alpha$, importin $\beta$ showed different proteolytic susceptibility. In the importin $\alpha /$ importin $\beta$ complex purified by gel filtration and digested with chymotrypsin under identical conditions, importin $\beta$ became resistant to proteolysis. As shown in Fig. 1a, lane 3, the digested complex migrated on SDS-PAGE as a single band, corresponding to the full length importin $\beta$. In contrast, the proteolytic behavior of importin $\alpha$ appeared unchanged by the interaction with importin $\beta$. In both cases the protein was severely digested by the protease (Fig. 1a, lanes 1 and 3). Addition of $500 \mathrm{mM}$ magnesium chloride to the importin $\alpha /$ importin $\beta$ heterodimer abolished the proteolytic resistance of importin $\beta$. Under these conditions, the complex was disrupted [17] and free importin $\beta$ was digested yielding the fragment 1-449 (Fig. 1a, lane 6). Thus, the proteolytic resistance of importin $\beta$ depended on the physical association with importin $\alpha$ and could be disrupted by increasing the ionic strength.

The digested importin $\alpha /$ importin $\beta$ complex was analyzed by non-denaturating gel electrophoresis and showed a clear shift in the electrophoretic mobility (Fig. 1b). This suggested a considerable change in shape and/or charge of the complex compared to undigested importin $\beta$. Analysis of the purified digested complex by mass spectrometry revealed the presence of a $5.3 \mathrm{kDa}$ peptide associated with importin $\beta$ matching the IBB domain of importin $\alpha$ (data not shown). The lower electrophoretic mobility (Fig. 1b, lanes 2 and 3 compared to lane 1) is likely caused by a change in charge due to the association of importin $\beta$ with the IBB domain, which bears a large positive (+9) net charge at neutral $\mathrm{pH}$. We then repeated the proteolysis of importin $\beta$ in the presence of chemically synthesized IBB domain peptide (residues $\alpha 11-\alpha 54$ of human importin $\alpha-2$ ). At an equimolar ratio of protein and IBB domain the proteolytic protection of importin $\beta$ was already established (Fig. 1c, lane 4), confirming that the IBB domain of importin $\alpha$ was sufficient to induce proteolytic protection of importin $\beta$ and that no other regions of importin $\alpha$ were required. The same proteolytic protection of importin $\beta$ was observed when the IBB domain of importin $\alpha$ was replaced by the adapter independent, IBB-like substrate HIV-1 Rev [18]. Fig. 1d, lanes 7-8, show that in an importin $\beta$ :Rev complex, importin $\beta$ remained uncleaved compared to free importin $\beta$ digested under the same conditions (lane 4).

Finally, we investigated the structural stability of importin $\beta$ in complex with RanGTP. Chymotryptic digestion of the RanGTP:importin $\beta$ complex only revealed weak proteolytic protection of importin $\beta$, which was again mainly cleaved at position 449, yielding fragment 1-449 (Fig. 1e, lanes 4-5). The digested importin $\beta$ (1-449):RanGTP complex was not disrupted by the cleavage and could be further isolated by gel filtration chromatography (data not shown), as the RanGTP binding domain lies within the first 10 HEAT repeats of importin $\beta$ [21,28]. Addition of RanBP1 [25] did not alter the proteolytic pattern of importin $\beta$ bound to RanGTP (Fig. 1e, lane 6 compared to lane 5).

\subsection{Binding of the IBB domain enhances the stability, increases the fluorescence emission and reduces the hydrodynamic radius of importin $\beta$}

We next investigated the stability of importin $\beta$ upon binding to the IBB domain of importin $\alpha$ by monitoring the thermal unfolding of the protein. Denaturing curves were recorded following variation of ellipticity at $222 \mathrm{~nm}$ as a function of the temperature. In the absence of the IBB domain, importin $\beta$ unfolded irreversibly as a single entity (Fig. 2a) with a $T_{\mathrm{m}}$ of about $49.5^{\circ} \mathrm{C}$. The slope of the sigmoidal transition between native and denatured states of the protein (correlated with the cooperativity of the process [27,29]) suggested that the denaturation of importin $\beta$ is highly cooperative. The addition of the IBB domain did not significantly change the slope of the melting curve but shifted $T_{\mathrm{m}}$ by approximately $10^{\circ} \mathrm{C}\left(T_{\mathrm{m}}=59^{\circ} \mathrm{C}\right)$. Thus, in accordance with the proteolytic resistance, binding of the IBB peptide caused a considerable stabilization of importin $\beta$.

Analysis of the fluorescence emission of importin $\beta$ is

Fig. 1. Proteolytic assay. a: Recombinant importin $\alpha$, importin $\beta$ and importin $\alpha / \beta$ complex were digested with chymotrypsin in $50 \mathrm{mM}$ sodium chloride (lanes 1,2 and 3) and $500 \mathrm{mM}$ magnesium chloride (lanes 4,5 and 6) Controls for undigested importin $\alpha$ and $\beta$ and importin $\alpha / \beta$ complex are shown in Fig. 3a, lane 1, in (c) (this figure), lane 1, and in (d) (this figure), lane 3, respectively. b: Importin $\alpha / \beta$ complex digested for 60 and 90 min (lanes 2 and 3, respectively) was separated on non-denaturing polyacrylamide gel using importin $\beta$ as control (lane 1). c: Limited proteolysis of importin $\beta$ in the presence of the IBB domain of importin $\alpha$. Proteolytic resistance of importin $\beta$ was already observed at a molar ratio of 1:1 (lane 4). d: HIV-1 Rev induces proteolytic resistance of importin $\beta$. The complex importin $\beta$ :Rev is still mostly uncleaved after 60 and $90 \mathrm{~min}$ of digestion (lanes 7 and 8, respectively). e: The RanGTP bound state of importin $\beta$ is sensitive to chymotrypsin cleavage at position 449 (lane 5). The addition of RanBP1 to the importin $\beta$ :RanGTP complex (lane 3 ) did not alter the proteolytic pattern of importin $\beta$ (lane 6). 
shown in Fig. 2b. Addition of the IBB domain stimulated the fluorescence emission of importin $\beta$ more than seven-fold (885 versus 125 FAU) and the maximum of emission was significantly blue-shifted (126 FAU at $342 \mathrm{~nm}$ versus 885 FAU at $335 \mathrm{~nm}$ ). In analogy with the fluorescence variations recorded during the folding of a polypeptide chain [27], tryptophan side chains are buried from the solvent and presumably protected from collisional quenching by water molecules in the importin $\beta$ :IBB domain complex. This agrees with the results of our crystallographic analysis, where several tryptophan residues

a

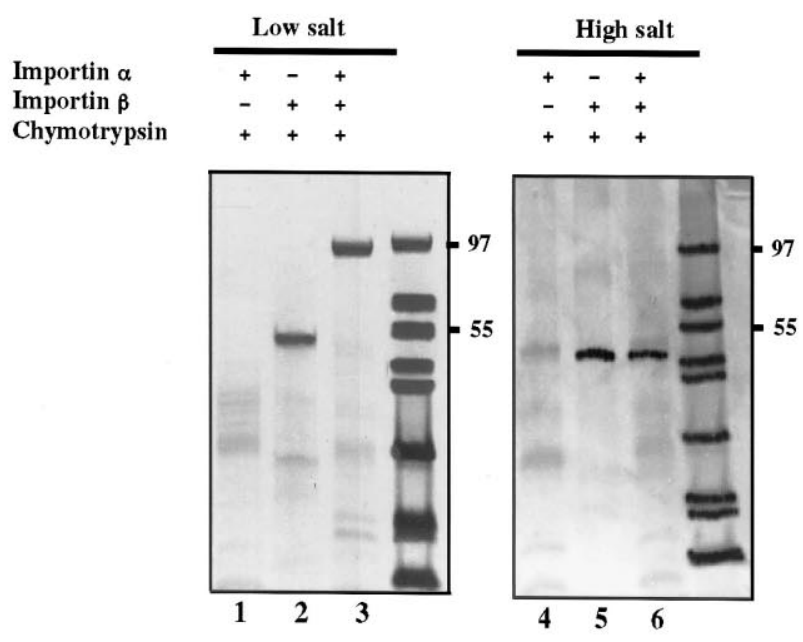

b
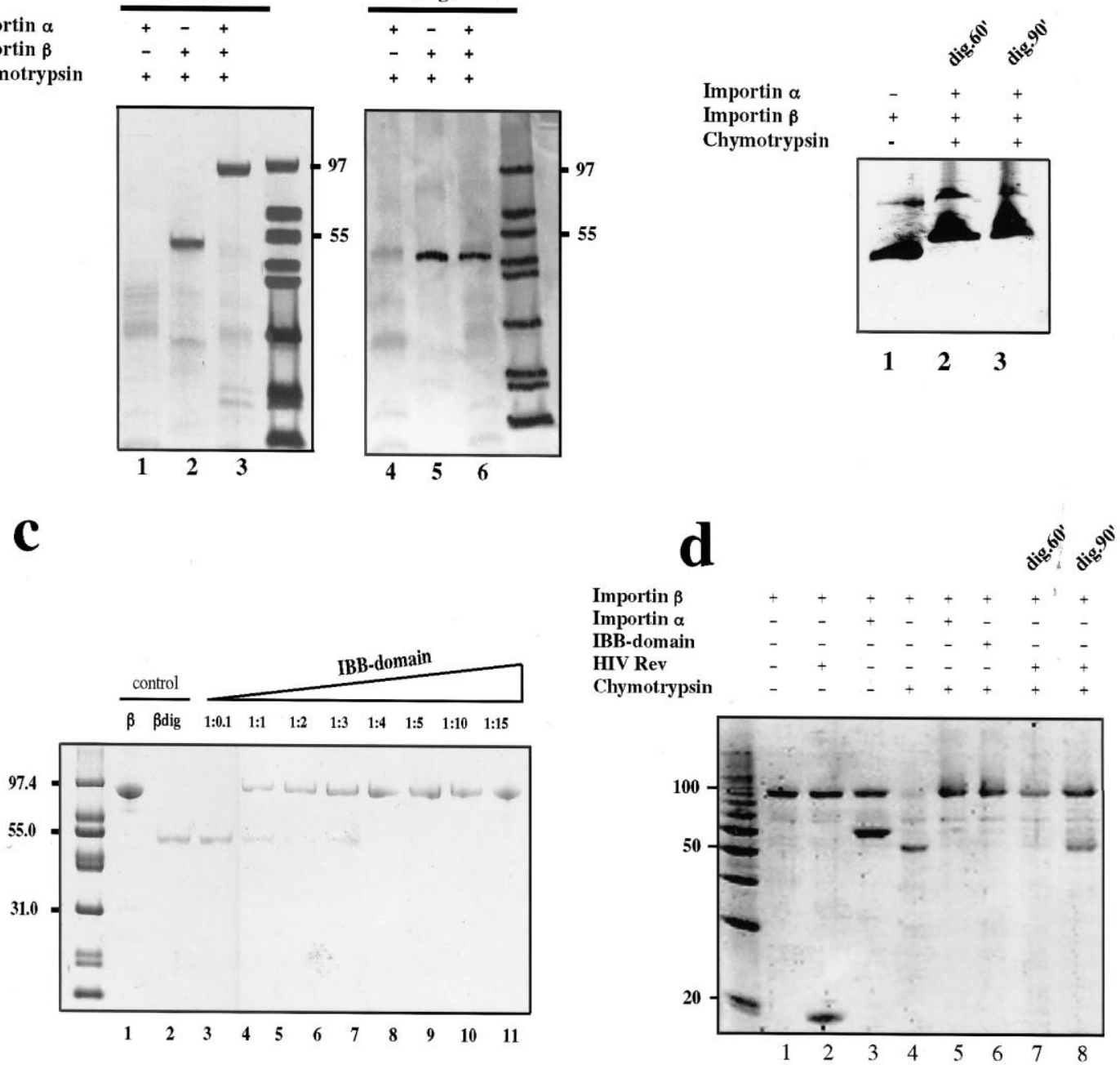

e

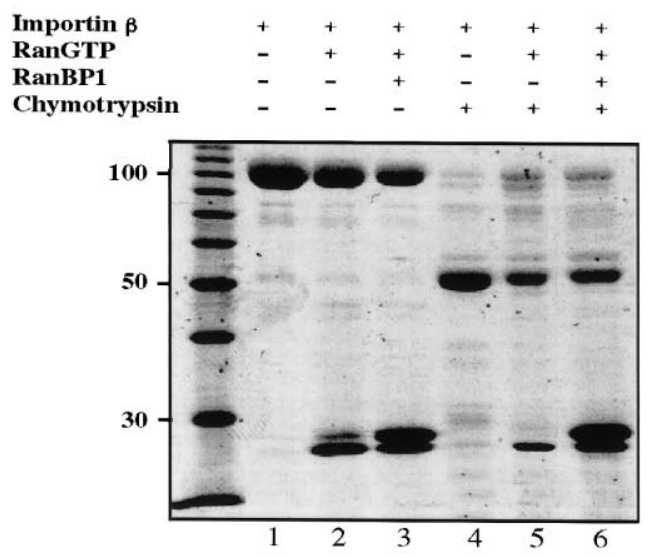


a

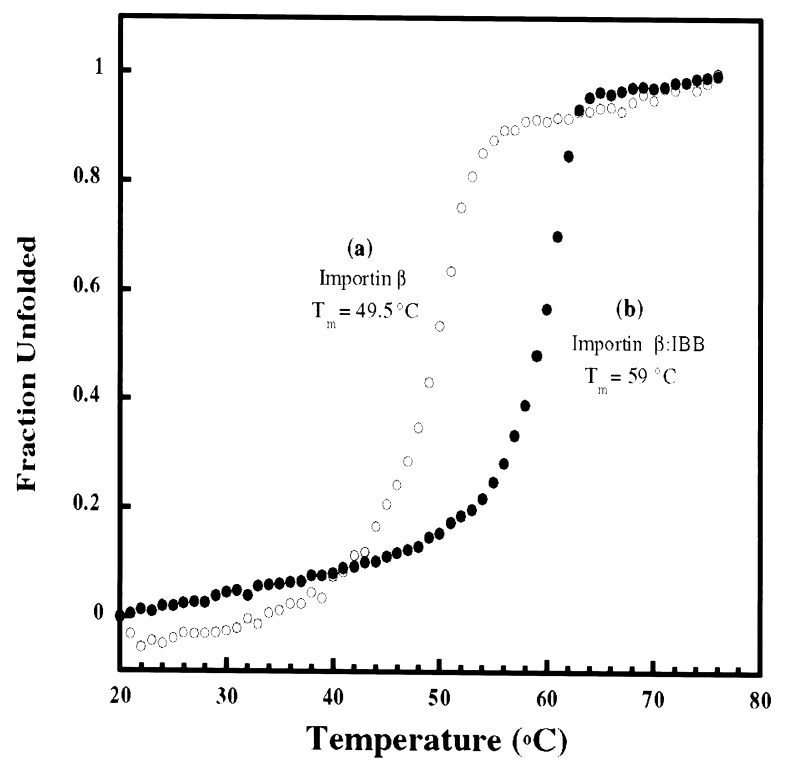

b

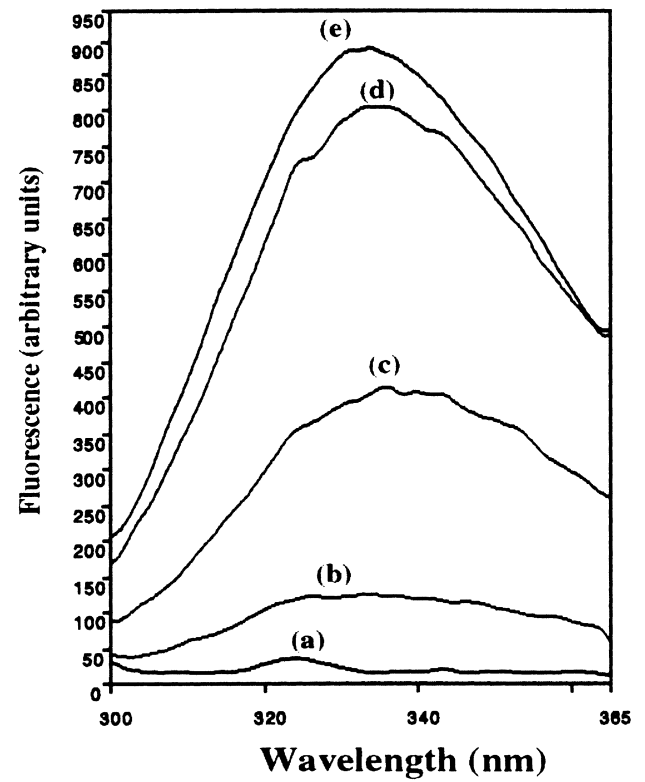

C

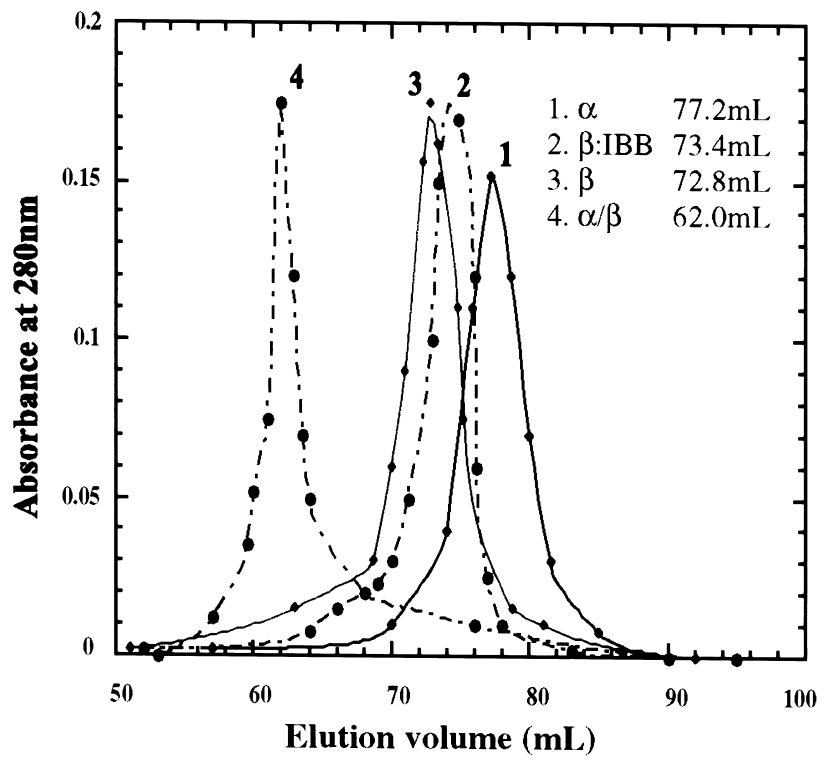

Fig. 2. Biophysical analysis. a: Thermal stability of importin $\beta$ in the presence and absence of the IBB domain. Changes in the mean ellipticity at $222 \mathrm{~nm}$ as a function of temperature for importin $\beta$ (curve a) and importin $\beta$ :IBB domain complex (curve b). b: Increase of the intrinsic tryptophan fluorescence as a result of the IBB domain binding. Curves a and b show the intrinsic emission of the IBB domain ( $\alpha 11-\alpha 54)$ and importin $\beta$, respectively. After addition of the IBB domain, fluorescence was measured immediately (curve c), after 1 min (curve d) and at equilibrium (curve e). c: Gel filtration elution profiles of importin $\alpha$, importin $\beta$ and the complexes importin $\beta$ :importin $\alpha$ and importin $\beta$ :IBB domain.

become buried in the IBB domain interface, when importin $\beta$ wraps around the IBB domain. Gel filtration chromatography also suggests a more compact conformation of importin $\beta$ when bound to the IBB peptide, despite of its larger mass (Fig. 2c). We found that the elution volume of the importin $\beta$ :IBB domain complex was slightly larger than free importin $\beta$ (73.4 ml versus $72.8 \mathrm{ml}$ ) which supported the idea that importin $\beta$ switches its conformation upon interaction with the IBB domain, adopting the globular shape observed in the crystal structure of the complex [20].

\subsection{Helix formation in the IBB domain}

Proteolysis under the conditions used for importin $\beta$ leads to the complete degradation of importin $\alpha$ (Fig. 1a, lane 1). Digestion of importin $\alpha$ under milder conditions resulted in cleavage between residues $\alpha 27$ and $\alpha 28$ and between $\alpha 34$ and $\alpha 35$ (Fig. 3a, lane 2) suggesting that this region of the IBB domain is not fully structured in solution. Similar results have been obtained for yeast importin $\alpha$ [12], where cleavage occurs after residue $\alpha 45$ (residue $\alpha 40$ human importin $\alpha$ numbering). In the crystal structure of mouse importin $\alpha$ IBB residues 
a

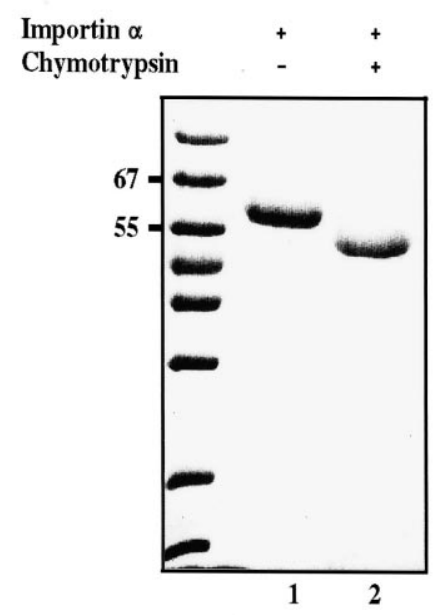

b

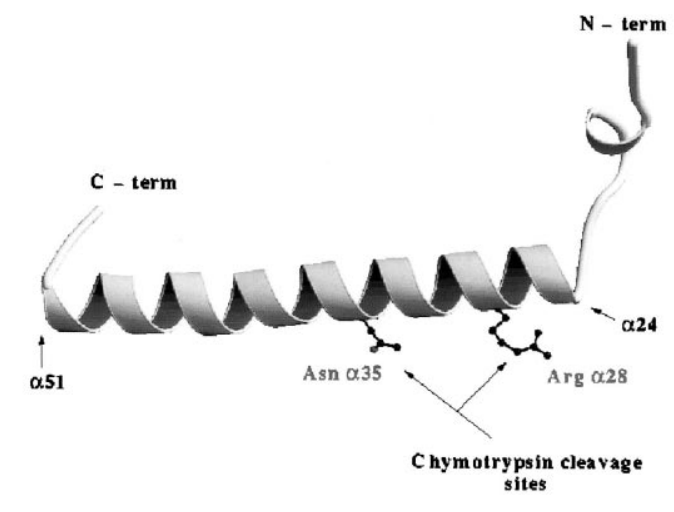

Circular dichroism spectra

c

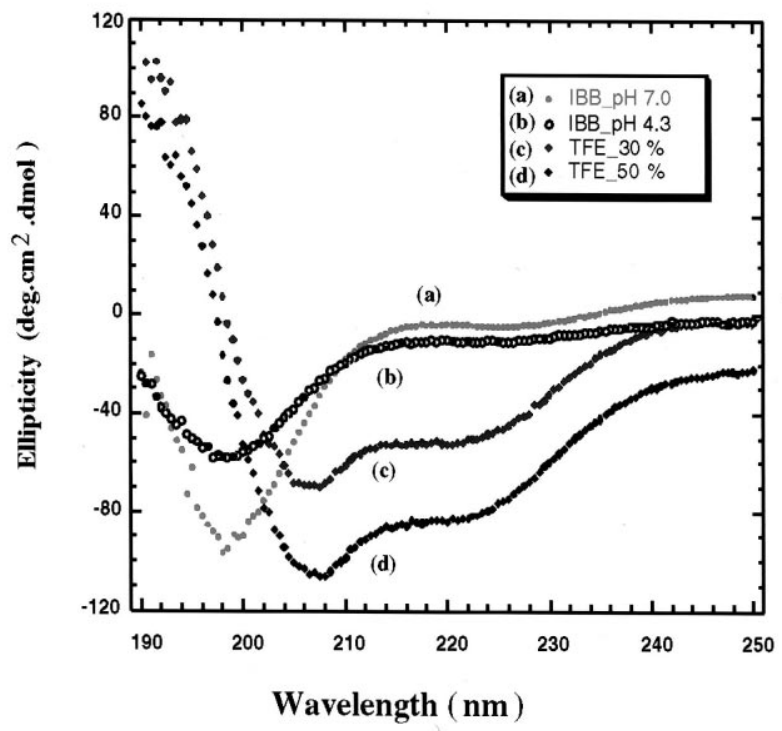

Fig. 3. Helix formation in the IBB domain. a: Limited proteolysis of importin $\alpha$ under mild digestion conditions. b: Ribbon representation of the IBB domain of importin $\alpha$ produced with programs Molscript [37] and RASTER3D [38]. c: CD analysis of the IBB domain. Spectra (a), (c) and (d) were recorded at $\mathrm{pH} 7.0$, while spectra (b) was recorded at $\mathrm{pH} 4.2$. The addition of TFE to a final concentration of $30 \%$ (c) and $50 \%$ (d) rapidly induced helix formation.

$\alpha 44-\alpha 54$ are bound in an extended conformation to the NLS binding site, which suggested an autoinhibitory mechanism for NLS-substrate binding [30]. These results are in contrast with the structure of the IBB domain bound to importin $\beta$ where residues $\alpha 24-\alpha 51$ form a long $\alpha$ helix (Fig. 3b) [20]. We used $\mathrm{CD}$ to investigate the secondary structure content of the IBB domain in solution. Spectra recorded at neutral (7.0) and acidic (4.2) $\mathrm{pH}$ showed that the IBB domain is predominantly unstructured in solution (Fig. 3c, curves a and b). $\alpha$ helix formation could be induced by the addition of 30\% TFE [26] (Fig. 3c, curves c and d) as revealed by a double minimum in the ellipticity $(\theta)$ value at 222 and $205 \mathrm{~nm}$ and a maximum at $195 \mathrm{~nm}$. The helical content in the presence of TFE was estimated to be about $70 \%$, which corresponds to the helix content seen in the crystal structure (27 out of 43 residues are helical, Fig. 3b). These data supported the hypothesis that the IBB domain of importin $\alpha(\alpha 24-\alpha 51)$ has an internal propensity to adopt a helical conformation, but that interaction with importin $\beta$ is required to adopt this conformation.

\section{Discussion}

\subsection{Proteolysis indicates a conformational change}

Limited proteolysis provides information about cleavage site accessibility and the flexibility of proteins in solution. Regions buried in the interior of the protein are protected from proteolysis, while solvent accessible, flexible regions are preferably cleaved by proteases. Changes in the susceptibility of proteins to proteolysis upon binding to interacting partners can be caused by steric hindrance protecting potential cleav- 


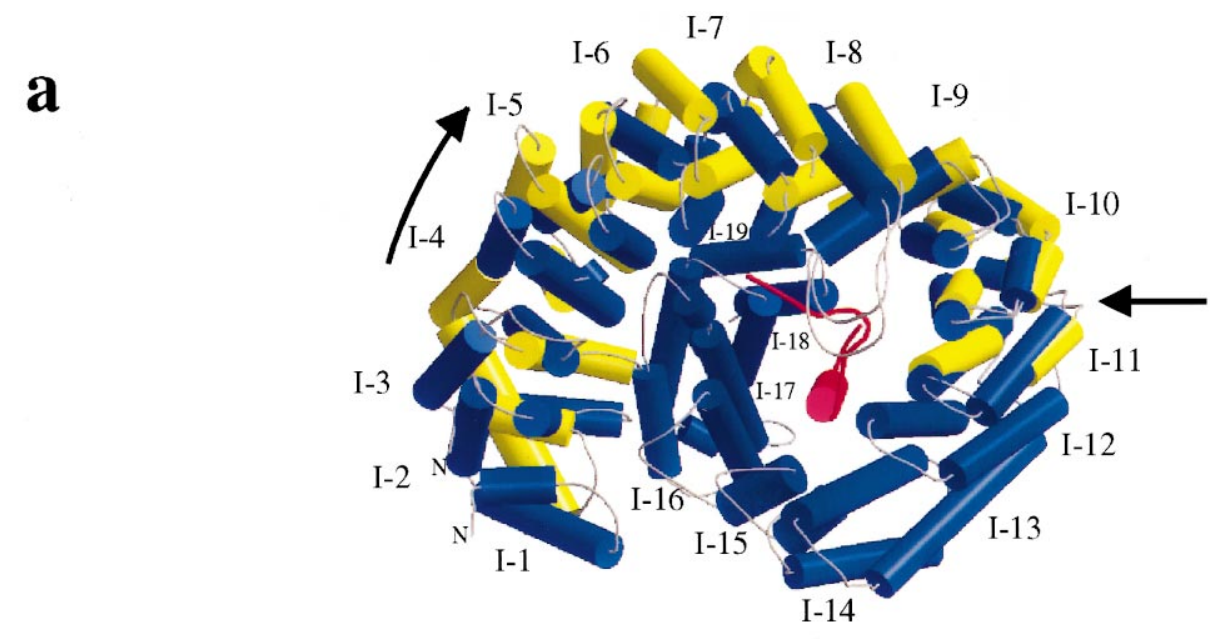

b

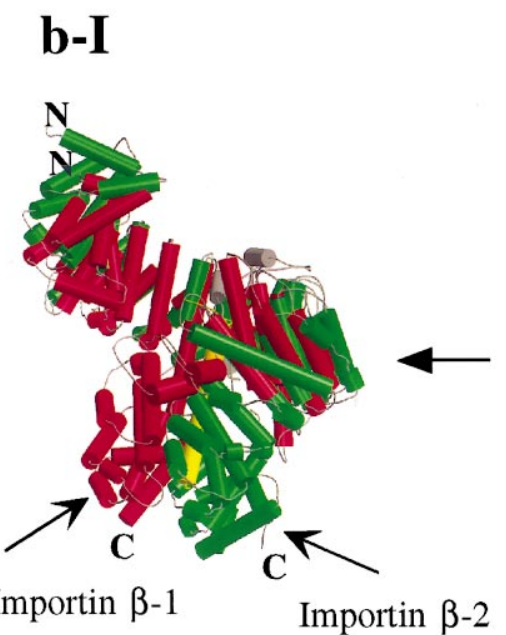

b-II

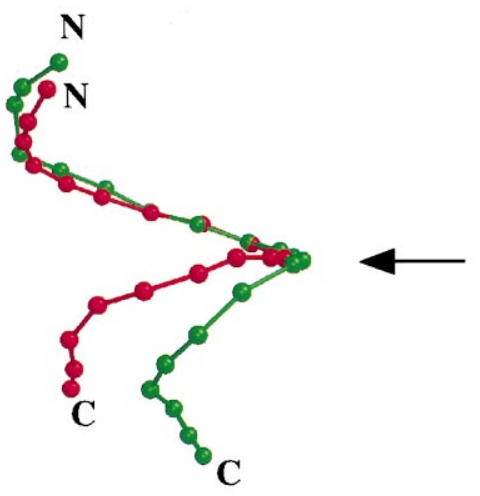

b-III

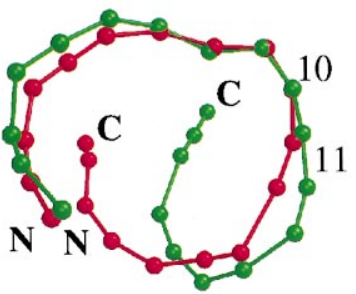

Fig. 4. a: Ribbon diagram of importin $\beta-1$ in crystal form I (blue) and repeats 1-11 in crystal form II (yellow). The IBB domain is depicted in red. Arrows indicate the chymotrypsin cleavage site and movement of the $11 \mathrm{~N}$-terminal repeats in crystal form II with respect to crystal form I. b-I: Ribbon diagram of importin $\beta$ (red) bound to the IBB domain (in yellow) superimposed with karyopherin $\beta-2$ (green). In both receptors $\mathrm{A}$ and $\mathrm{B}$ helices connecting residues are depicted in gray. Two orthogonal views are given in (b-II) and (b-III), where HEAT repeats of importin $\beta$ and karyopherin $\beta-2$ are depicted as red and green spheres. The chymotrypsin cleavage site is indicated by an arrow. The figure was produced with programs Molscript [37] and RASTER3D [38].

age sites from proteolysis but can also indicate conformational changes [31-33].

The chymotrypsin cleavage site observed in free importin $\beta$ can be mapped onto the crystal structures of importin $\beta$ bound to the IBB domain complex (Fig. 4a, [20]) and onto one of the molecules (residue 1-459) of the N-terminal importin $\beta$ fragment bound to RanGTP [21]. In the second molecule of the importin $\beta$ fragment:RanGTP complex present in the asymmetric unit the chain is disordered after residue 439 [21], whereas the N-terminal importin $\beta$ construct used in the crystal structure of the FxFG nucleoporin complex ends after residue 442 [22]. The cleavage site is located at the $\mathrm{N}$-terminal end of helix A of HEAT-11, such that the chymotryptic fragment 1-449 includes the N-terminal 10 HEAT motifs. Residue 449 is a leucine residue, which corresponds to the specificity of chymotrypsin for large hydrophobic residues.

The chymotrypsin cleavage site is located at the outer surface of importin $\beta$ (exclusively formed by helices $A$ ) whereas the IBB domain binds the inner surface of importin $\beta$ (exclu- sively formed by helices B). Therefore, the increased proteolytic resistance of importin $\beta$ in complex with the IBB domain compared to free importin $\beta$ and importin $\beta$ bound to RanGTP cannot be simply explained by the reduced accessibility of the cleavage site due to 'steric hindrance' by the peptide binding. We rather conclude that free importin $\beta$ and importin $\beta$ bound to RanGTP (or RanGTP:RanBP1) adopt similar conformations sensitive to chymotrypsin whereas binding of the IBB domain leads to a different proteolytically resistant conformation. A conformational change in importin $\beta$ upon binding of the IBB domain is also consistent with our results from size exclusion chromatography, thermal unfolding analysis and fluorescence spectroscopy.

\subsection{Structural evidence for conformational changes}

Correlating our solution studies with the available X-ray structures provides further support for a conformational change between unbound and Ran:GTP-bound importin $\beta$ and importin $\beta$ bound to the IBB domain: in the N-terminal 
importin $\beta$ fragment bound to RanGTP helix A of repeat 11 has shifted by about $8 \AA$ away from the core of the molecule compared to its position observed in the importin $\beta$ :IBB complex. Similarly in the crystal structure of the homologous import receptor karyopherin $\beta 2$ (importin $\beta 2$, transportin) bound to RanGTP [23] karyopherin $\beta 2$ adopts a more open conformation with its $\mathrm{C}$-terminal moiety being rotated by almost $30^{\circ}$ away from its $\mathrm{N}$-terminal half (Fig. 4b). Because this rotation also involves incremental rearrangements between individual repeats and because of intrinsic differences between importin $\beta$ and karyopherin $\beta 2$, a 'hinge region' cannot be unambiguously assigned. However, the proteolytic cleavage site coincides with the region where polypeptide chains of karyopherin $\beta 2$ and importin $\beta$ start to deviate (Fig. 4b). Intrinsic flexibility in this area of the importin $\beta$ superhelix is also suggested by two slightly different crystal forms of the importin $\beta$ :IBB complex, where $\mathrm{N}$-terminal repeats $1-11$ and C-terminal repeats $12-19$ rotate $10^{\circ}$ with respect to each other (Fig. 4a). In a model of the importin $\beta$ :RanGTP complex based on the structure of full length importin $\beta$ bound to the IBB domain superimposed on the structure of the $\mathrm{N}$-terminal fragment bound to RanGTP, steric clashes occur between parts of Ran and HEAT repeats 13-14. To avoid these clashes importin $\beta$ has to adopt a more open conformation when it accommodates RanGTP.

Binding of RanBP1 does not change the proteolytic sensitivity of importin $\beta$ bound to RanGTP. This is not surprising because in a model of a ternary importin $\beta$ :RanGTP: RanBD1 complex based on the co-crystal structures of RanGppNHp bound to the first 10 HEAT repeats of importin $\beta$ [21] and to the RanBP1-like Ran binding domain of RanBP2 [34], RanBD1 does not contact importin $\beta$ directly.

\subsection{Conformational changes in nuclear transport}

Our results using limited proteolysis, fluorescence spectroscopy and $C D$ suggest binding-induced conformational changes in importin $\alpha$ and importin $\beta$. Accordingly importin $\beta$ exists in at least two different conformational states (Fig. 5a): an open conformation with high affinity for the IBB substrate, and a closed conformation when bound to the IBB domain of an import substrate. The transition from the open to the closed conformation can be induced by the IBB domain of importin $\alpha$, as well as by other IBB-like substrates, like HIV-1 Rev. Binding of RanGTP is incompatible with a closed conformation of importin $\beta$ as clashing would occur between the two proteins. The mechanism by which RanGTP induces the opening of importin $\beta$ remains elusive. However, it is noteworthy that residues $124-140$ of RanGTP in the importin $\beta$ :RanGTP complex occupy a binding region previously occupied by IBB domain residues. In particular the side chain of Arg140 in RanGTP substitutes for $\alpha$ Arg13 of the IBB domain by making very similar interactions with importin $\beta$. A sequential release of the IBB domain could be initiated at this position.

Concomitantly to the tertiary structure rearrangements in importin $\beta$, the IBB domain also undergoes a conformational change (Fig. 5b). It adopts a helical fold when bound to importin $\beta$, while in solution the IBB domain is essentially unstructured, except for a small region, which interacts with the NLS binding region of importin $\alpha$, as shown in the structure of mouse importin $\alpha$ [30]. A similar structural plasticity has been observed for the HIV-1 Rev protein: an arginine- a

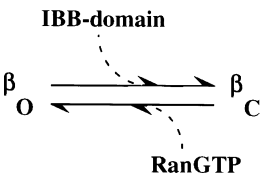

Fig. 5. NLS dependent nuclear import is mediated by double conformational switch of importin $\alpha$ and $\beta$. a, b: Schematic diagram of the importin $\beta\left(\beta_{\mathrm{C}}=\right.$ closed, $\beta_{\mathrm{O}}=$ open $)$ and IBB domain conformers.

rich Rev peptide (residues 34-50) is fully helical in complex with a 35-mer RRE RNA aptamer I [35], whereas it adopts an extended conformation in complex with the 27-mer RRE RNA aptamer II [36]. The transition between the two structural forms presumably occurs upon interaction with the acidic RNA pocket. The same region of HIV-1 Rev is involved in the direct binding to importin $\beta[18]$ and can also induce proteolytic resistance of importin $\beta$ (Fig. 1d) suggesting that HIV-1 Rev functions similarly to the IBB domain and is also able to induce a conformational change.

Conformational changes in importin $\beta$ are not only important for binding and release of import substrates as discussed above but also for its interactions with nucleoporins. Comparison of the $\mathrm{N}$-terminal importin $\beta$ fragment structures bound to RanGTP and to nucleoporin reveals movements between $\mathrm{A}$ helices in repeat 5 and 6 , which could provide a structural explanation for how RanGTP binding displaces importin $\beta$ from FxFG nucleoporin repeats [22]. Functional conformational flexibility of importin $\beta$ therefore appears to be a reoccurring theme and is expected to be observed in the entire importin $\beta$ superfamily [5]. All family members are predicted to share a similar HEAT repeated architecture as observed in importin $\beta$ and karyopherin $\beta 2$. The stacking of individual repeats results in elongated rod-like structures. Interactions with transport substrates are predicted to induce conformational changes of these elongated molecules, which are counteracted or enhanced by RanGTP for import and export substrates, respectively. Furthermore, conformational changes could also increase or decrease the affinities of nuclear transport receptors to the different components of the nuclear pore which would provide a structural basis for the mechanism of their translocation.

Acknowledgements: We thank John Johnson at the Scripps Research Institute and Carlo Petosa and Florence Baudin at the EMBL-Outstation, Grenoble for helpful discussions and critical reading of the manuscript. G.C. acknowledges support by the EMBL international Ph.D. Program and a 'Human Frontier Science Program' post-doctoral fellowship.

\section{References}

[1] Mattaj, I.W. and Englmeier, L. (1998) Annu. Rev. Biochem. 67, 265-306.

[2] Görlich, D. and Kutay, U. (1999) Annu. Rev. Cell Dev. Biol. 15 , 607-660.

[3] Melchior, F., Paschal, B., Evans, J. and Gerace, L. (1993) J. Cell Biol. 123, 1649-1659.

[4] Moore, P.A., Ruben, S.M. and Rosen, C.A. (1993) Mol. Cell. Biol. 13, 1666-1674.

[5] Görlich, D., Dabrowski, M., Bischoff, F.R., Kutay, U., Bork, P., Hartmann, E., Prehn, S. and Izaurralde, E. (1997) J. Cell Biol. $138,65-80$. 
[6] Görlich, D., Kostka, S., Kraft, R., Dingwall, C., Laskey, R.A., Hartmann, E. and Prehn, S. (1995) Curr. Biol. 5, 383-392.

[7] Chi, N.C., Adam, E.J.H. and Adam, S.A. (1995) J. Cell Biol. $130,265-274$.

[8] Radu, A., Blobel, G. and Moore, M.S. (1995) Proc. Natl. Acad. Sci. USA 92, 1769-1773.

[9] Görlich, D., Prehn, S., Laskey, R.A. and Hartmann, E. (1994) Cell 79, 767-778.

[10] Weis, K., Mattaj, I.W. and Lamond, A.I. (1995) Science 268, $1049-1053$.

[11] Moroianu, J., Blobel, G. and Radu, A. (1995) Proc. Natl. Acad. Sci. USA 92, 2008-2011.

[12] Conti, E., Uy, M., Leighton, L., Blobel, G. and Kuriyan, J. (1998) Cell 94, 193-204.

[13] Huber, J., Cronshagen, U., Kadokura, M., Marshallsay, C., Wada, T., Sekine, M. and Lührmann, R. (1998) EMBO J. 17, $4114-4126$.

[14] Jäkel, S., Albig, W., Kutay, U., Bischoff, F.R., Schwamborn, K., Doenecke, D. and Görlich, D. (1999) EMBO J. 18, 2411-2423.

[15] Jullien, D., Görlich, D., Laemmli, U.K. and Adachi, Y. (1999) EMBO J. 18, 4348-4358.

[16] Görlich, D., Panté, N., Kutay, U., Aebi, U. and Bischoff, F.R. (1996) EMBO J. 15, 5584-5594.

[17] Weis, K., Ryder, U. and Lamond, A.I. (1996) EMBO J. 15, $1818-1825$.

[18] Truant, R. and Cullen, B.R. (1999) Mol. Cell. Biol. 19, 12101217.

[19] Jäkel, S. and Görlich, D. (1998) EMBO J. 17, 4491-4502.

[20] Cingolani, G., Petosa, C., Weis, K. and Müller, C.W. (1999) Nature 399, 221-229.

[21] Vetter, I.R., Arndt, A., Kutay, U., Görlich, D. and Wittinghofer, A. (1999) Cell 97, 635-646.
[22] Bayliss, R., Littlewood, T. and Stewart, M. (2000) Cell 102, 99108.

[23] Chook, Y.M. and Blobel, G. (1999) Nature 399, 230-237.

[24] Groves, M.R., Hanlon, N., Turowski, P., Hemmings, B.A. and Barford, D. (1999) Cell 96, 99-110.

[25] Coutavas, E., Ren, M., Oppenheim, J.D., D’Eustachio, P. and Rush, M.G. (1993) Nature 366, 585-587.

[26] Kumaran, S. and Roy, R.P. (1999) J. Pept. Res. 53, 284-293.

[27] Pace, C.N., Scholtz, J.M. and Creighton, T.E. (1997) Protein Structure: a Practical Approach, pp. 299-321.

[28] Kutay, U., Izaurralde, E., Bischoff, F.R., Mattaj, I.W. and Görlich, D. (1997) EMBO J. 16, 1153-1163.

[29] Kelly, S.M. and Price, N.C. (1997) Biochim. Biophys. Acta 1338, 161-185.

[30] Kobe, B. (1999) Nat. Struct. Biol. 6, 388-397.

[31] Hubbard, S.J., Eisenmenger, F. and Thornton, J.M. (1994) Prot. Sci. 3, 757-768.

[32] Cohen, S.L., Ferre-D'Amare, A.R., Burley, S.K. and Chait, B.T. (1995) Prot. Sci. 4, 1088-1099.

[33] Orru, S., Dal Piaz, F., Casbarra, A., Biasiol, G., De Francesco, R., Steinkuhler, C. and Pucci, P. (1999) Prot. Sci. 8, 1445-1454.

[34] Vetter, I.R., Nowak, C., Nishimoto, T., Kühlmann, J. and Wittinghofer, A. (1999) Nature 398, 39-46.

[35] Ye, X., Gorin, A., Ellington, A.D. and Patel, D.J. (1996) Nat. Struct. Biol. 3, 1026-1033.

[36] Ye, X. et al. (1999) Chem. Biol. 6, 657-669.

[37] Kraulis, P.E. (1991) J. Appl. Crystallogr. 24, 946-950.

[38] Merritt, E.A. and Bacon, D.J. (1997) Methods Enzymol. 277, $505-524$. 